\title{
Personalised medicine approaches to screening and prevention
}

\section{Kezia Gaitskell}

Nuffield Department of Population Health, University of Oxford, UK, and The Royal Free Hospital, London

Email: kezia.gaitskell@ceu.ox.ac.uk

Funding details: None

Disclosure statement: No conflicts of interest to disclose.

Biographical notes: Kezia Gaitskell is a specialty registrar in Histopathology, and has recently completed a DPhil in Population Health at the University of Oxford, funded by Cancer Research UK. Correspondence to: kezia.gaitskell@ceu.ox.ac.uk. 


\section{Personalised medicine approaches to screening and prevention}

One of the promised benefits of 'personalised' medicine is that it will have a positive impact on disease screening and prevention, by enabling more person-specific estimates of risk, and hence more personalised strategies for screening and risk reduction. This article summarises some of the areas in which these approaches are already being implemented, but also outlines some of the potential difficulties and limitations - and challenges the assumption that 'personalised' approaches are necessarily superior for prevention and screening.

Keywords: Personalised medicine, screening, prevention, risk reduction, genetic testing, epidemiology

\section{Introduction}

Several authors have emphasised that the new personalised or '4P' medicine should be 'predictive' and 'pre-emptive' (Horne et al. 2015), or 'preventive' (Flores et al. 2013) (as well as 'personalised' and 'participatory'). This tends to have two broad aspects: 1) the incorporation of 'personalised' information into screening programmes, so as to enable screening to be more targeted than general population-based approaches, and 2) the potential impact of personalised information regarding risk to facilitate personalised risk-reduction strategies (e.g. in terms of lifestyle choices or drug therapy).

This paper summarises some of the hoped-for promise of these more 'personalised' strategies for disease screening and prevention, and outlines existing areas of implementation, but also discusses some of the challenges encountered, and the limitations relative to more conventional methodologies. The examples discussed are mostly from the field of cancer screening and prevention, as much of the research thus far has focussed on this area - but many of the same principles would also apply to screening and prevention for other diseases. 


\section{Personalised Screening}

\section{The promise of personalised screening}

The aim with any screening programme is to detect abnormalities earlier than patients would notice changes themselves (symptoms). For cancer screening, this may either be early-stage cancer or precancerous changes - as treatments tend to be more effective at earlier stages. Screening generally involves a two-phase testing process, in which an initial 'screening' test is applied to the whole population (often with age or gender limits, depending on the disease), which identifies people at higher risk of the disease, who then have a second 'diagnostic' test. The screening test is usually low-risk and relatively inexpensive (e.g. a mammogram in breast cancer screening), whereas the diagnostic test may have slightly higher costs, both financial and risk or inconvenience (e.g. taking a tissue biopsy).

No test is perfect, and so there will be both 'false positives' (people who test positive, but do not have the disease), and 'false negatives' (people who test negative, but do in fact have the disease). Another important concept in screening is that of 'over-diagnosis' - i.e. of diagnosing a cancer that would never have been detected in a person's lifetime without the use of screening, as they would have died of other causes before the cancer became symptomatic. As a diagnosis of cancer is not only psychologically upsetting, but also entails all the potential risks of treatment (e.g. surgery, chemotherapy, or radiotherapy), any screening programme aims to limit 'over-diagnosis' and 'over-treatment', whilst also detecting the cancers that would genuinely cause problems if not diagnosed and treated.

One of the hopes of 'personalised' approaches to screening is that the incorporation of person-specific information (e.g. inherited genetic mutations) will give more nuanced estimates of risk. This might be helpful both in terms of targeting screening at specific populations and modifying screening protocols (e.g. screening high-risk people from a 
younger age, and more frequently), and could also potentially lead to reduced screening for those at lower risk. Such 'personalised' screening could thus be better at detecting cancer in people who genuinely have it, whilst also reducing over-diagnosis.

\section{Personalised screening - current practice}

The cervical cancer screening programme in England is already incorporating molecular information into the screening process, by testing for high-risk strains of human papilloma virus (HPV, which causes almost all cases of cervical cancer) (Freeman-Wange et al. 2016). As women without high-risk HPV are very unlikely to have cervical pre-cancer, this is a useful initial triage step, and helps to reduce unnecessary further investigation of women without high-risk HPV. However, in this example the 'personalised' information is about what infections a person carries, not their own DNA.

Bowel cancer screening also already makes use of 'personalised' genetic risk estimates to an extent - in that people with known inherited genetic mutations that increase the risk of bowel cancer (e.g. FAP - familial adenomatous polyposis, or Lynch syndrome) are offered screening with colonoscopy from a younger age and more frequently than in the routine screening programme (Cairns et al. 2010). However, currently, testing for these inherited cancer syndromes tends to be limited to those with a strong family history of such cancers - and so other people with the mutations are probably being missed.

Outside of the cancer field, 'personalised' molecular information is already used in pre-conception screening for conditions such as Down's syndrome. The current means of screening for Down's syndrome in the UK usually involves a combination of a blood test for maternal hormone levels and an ultrasound of the foetus. However, a new form of screening test (known as non-invasive pre-natal testing, NIPT) makes use of small amounts of foetal DNA in the maternal blood, giving more accurate results. NIPT is not yet available as 
standard on the National Health Service in the UK (pending further evaluation), but is already used in the private sector (The NHS RAPID Project 2016).

\section{The challenges of personalised screening}

One of the challenges of targeted 'personalised' screening is that even if we can classify people at higher risk for a disease (e.g. by genetic testing), we still need an accurate screening test to identify people with the disease early enough to benefit them, and without harming too many people by 'over-diagnosing' (and 'over-treating') a 'disease' that might never have caused them problems.

These challenges partly explain the absence of national screening programmes for ovarian cancer and prostate cancer in the UK. Although we know that women with inherited mutations in the genes $B R C A 1$ and $B R C A 2$ are at increased risk of ovarian cancer (and breast cancer), we do not yet have clear evidence from randomised controlled trials that screening for ovarian cancer does more good than harm in either high-risk women or the general population (Jacobs et al. 2016; Rosenthal et al. 2013).

Similarly, some experts argue that there is insufficient evidence that prostate cancer screening (using a blood test for PSA, prostate-specific antigen) is accurate enough to do more good than harm, especially given the potential serious complications of treating prostate cancer (PDQ Screening and Prevention Editorial Board 2016). There have been several attempts to improve prostate cancer screening by stratifying men into risk categories using a combination of age and genetic information (as restricting screening to men at moderate to high risk should both reduce the number of people you need to screen, and reduce the number

of men 'over-diagnosed', without missing too many real cases) (Pashayan et al. 2015a; Pashayan et al. 2015b) - but these models are all based on extensive assumptions, and none have yet been trialled. 
However, this more stratified approach to screening is not without challenges - quite beyond the purely technical (though by no means trivial) question of whether we can accurately measure or predict the genetic risk of cancer for an individual or a population. In particular, stratifying for screening will lead to a substantial proportion of people not getting screening. While this may lead to good overall (screening low-risk people exposes them to all the harms of potential over-diagnosis, with less of the benefit of diagnosing and treating an otherwisefatal cancer), it will nevertheless lead to harm in some cases (some low-risk people will get aggressive cancer, which might have been picked up earlier if they had been screened). This may lead to anger from some people stratified into the 'low-risk'/ 'no screening' group. ${ }^{1}$

For those who are diagnosed with cancer by screening, there is also the challenge of knowing what to do with the information. For example, for clinically-localised prostate cancer detected by screening (rather than symptoms), it is arguable whether aggressive treatment with surgery or radiotherapy (both of which have significant risks of morbidity, including impotence and incontinence) are superior to watchful waiting (Hamdy et al. 2016).

The undesirability of screening or testing for conditions in the absence of a clearly defined therapy has also been raised regarding NIPT and other pre-conception screening, for which there may be few interventions available, other than termination - leading many people to express caution over the proposed introduction of NIPT, including in the popular press and social media (Groskop 2016). Similarly, in response to suggestions that newborns should undergo whole-genome sequencing, paediatricians, geneticists and ethicists have argued that newborn screening should be limited to the "targeted analysis and identification of gene variants conferring a high risk of preventable or treatable conditions, for which

\footnotetext{
${ }^{1}$ Though this challenge is not specific to genetic stratification: there was considerable anger expressed after the lower age limit of cervical cancer screening in the UK was raised from 20 to 25 in 2003 - a decision based on evidence that screening young women on balance did more harm than good, even though some young women with cancer would thereby not be diagnosed.
} 
treatment has to start in the newborn period or in early childhood" (Howard et al. 2015). A trial is ongoing in the USA to explore whether such sequencing in newborns does more good than harm (Berg et al. 2017).

There is also the issue of cost - 'personalised' medicine tends to involve molecular/ genetic testing, which is currently still relatively expensive compared to many existing screening tests. The new methods would have to lead to substantial savings in other ways (e.g. reducing over-diagnosis and expensive over-treatment, or reducing the number of people screened) in order to be cost-neutral.

\section{Personalised risk reduction based on personal knowledge}

\section{The promise of personalised risk reduction}

There is a hope that knowledge of personal risk (particularly through genetic/ genomic sequencing) will enable people to feel more empowered to act in ways to reduce that risk whether through specific medical interventions, or general lifestyle modifications (23andMe Inc. 2017). There has also been a suggestion by some (especially commercial companies) that sequencing at birth could help to identify many disease risks very early in life, allowing appropriate action (Human Longevity Inc. 2016).

\section{Personalised risk reduction - current practice}

As with 'personalised' screening, there is an extent to which 'personalised' risk reduction is already part of routine practice - but it tends to apply to selected groups, rather than the general population.

For example, people who are known to have an inherited genetic mutation causing raised blood cholesterol levels (familial hypercholesterolaemia) are often treated with 
cholesterol-lowering drugs (statins), to reduce their risk of cardiovascular disease (National Institite for Health and Care Excellence 2008 (Updated 2016)). However, as with the inherited cancer syndromes, we currently only tend to test people for these mutations if we know that they have a relative with the specific genetic mutation. Arguably more widespread testing would diagnose other people who might benefit (though at a significant cost).

Risk-reduction strategies are also advised for some people with the inherited colon cancer syndromes, and for women with inherited breast and ovarian cancer syndrome, in the rather drastic form of prophylactic surgery (total colectomy for some people with FAP and Lynch syndrome, or bilateral salpingo-oophorectomy and mastectomy for some women with BRCA1/2 mutations) (Paluch-Shimon et al. 2016; Stoffel et al. 2015). However, even this relatively long-standing advice is not straightforward - a recent study did not find a significant reduction in the risk of incident breast cancer amongst women with BRCA1 mutations who had bilateral oophorectomy (Kotsopoulos et al. 2017). The authors acknowledge that this is at odds with evidence from several other studies - but this apparent inconsistency merely highlights the complexity of obtaining reliable estimates of the potential impact of risk-reducing strategies. If it is this complex for a high-penetrance, high-risk genetic mutation that has been known about and tested for over many years, this suggests that it will be even more complicated for the combinations of common, small-magnitude, risk alleles that are likely to be at issue in general.

However, the existing interventions described above are for people known to be at very high risk - so the intervention is likely to be worthwhile (even if it involves major surgery). By contrast, attempts to estimate and modify risk in the general population tend to be based on multiple common genetic variants, each of which confers relatively modest increases in risk - and so the potential benefits are less clear. In addition, in these examples, 
the genetic risk is sufficiently high that people have often seen family members die of the disease, so the knowledge is not just a 'theoretical' risk based on a test result. It would require a further study to determine whether the experience of that sad circumstance would make acting on information about risk easier.

\section{The challenges of personalised risk reduction}

Unfortunately, the current evidence for personalised risk reduction is not very promising. A recent systematic review of 18 trials suggested that knowing about genetic risk did not lead people to behave in such a way as to reduce that risk (Hollands et al. 2016), as discussed by Joshua Hordern in more detail elsewhere in this issue. However, it highlights the point that, as with all information in medicine, it is generally only useful if we know what to do about it. There is arguably still some value in helping people to know about their personal risk prognosis (for planning purposes) - but mostly, the hope with personalised medicine is that it will not just be informative, but actionable. And for that, we come up against issues that people in public health have been battling with for years - how do you ensure effective, longterm, behaviour change, of a magnitude that will have a significant beneficial impact on health? We already know that lifestyle behaviours such as not smoking, avoiding obesity or malnourishment, having a balanced diet, and regular physical activity, would have a beneficial effect on disease prevention for almost everyone, regardless of personalised risk. The question of how to get people to act on this knowledge is considerably more complicated - to date there is no evidence that this can be helped by detailed molecular knowledge.

The Global Burden of Disease Study estimates that the ten leading risk factors for global morbidity and mortality are high systolic blood pressure, smoking, high fasting plasma glucose (i.e. diabetes and pre-diabetes), high BMI (body-mass index), childhood undernutrition, ambient particulate matter (i.e. outdoor air pollution), high total cholesterol, 
household air pollution, alcohol use, and diets high in sodium (GBD 2015 Risk Factors Collaborators 2016). Most, if not all, of these risk factors could be tackled by a combination of behaviour change (e.g. avoiding smoking and excess alcohol, maintaining a healthy BMI, diet modification, and physical activity), or relatively inexpensive generic drugs (e.g. antihypertensives to lower blood pressure, and statins to lower cholesterol). The evidence for the potential impact of modifying these risk factors is strong (Collins et al. 2016; Di Angelantonio et al. 2016; Norheim et al. 2015; Pirie et al. 2013).

For many low and middle income countries, substantial reductions in premature deaths could also be achieved by prevention or treatment of communicable diseases (e.g. tuberculosis, HIV, or malaria) and reduction of maternal and child mortality, using alreadyknown methods that do not require 'personalised' knowledge (e.g. immunisation for vaccinepreventable diseases, HIV treatment and prevention, insecticide-treated bed-nets to reduce malaria transmission, or oral rehydration therapy to reduce deaths of children with diarrhoeal diseases) (Norheim et al. 2015).

This strong evidence for simple, modifiable, risk factors is in stark contrast to the rhetoric of some of the private companies which offer personalised information on genetic risk. Such claims are made despite the fact that our knowledge of what many of these mutations mean in terms of risk often has a very limited evidence base, and it is often not clear what to do in terms of risk-reducing actions.

\section{Conclusion}

As described above, 'personalised' molecular approaches are already being applied to both screening and prevention in current clinical practice - but only in a few specific cases. The implication from some advocates of personalised medicine is that the same principles can 
apply to many or all diseases - and the evidence for this is far less clear. The examples above illustrate that, in each case, if we want to be personalised and preventive, we really need to understand the underlying biology. Simply sequencing DNA is unlikely to be helpful on its own - the clinical utility all depends on us knowing the significance of what we find.

Our understanding of the genetic and molecular determinants of disease has advanced significantly in a short period of time, and we hope that as we know more of what different mutations mean in terms of risk, we will also be able to develop strategies/ treatments to modify the risk. However, at the moment, there is the danger that some people are promising more than can in any way be delivered - and in the process, ignoring the knowledge we already have about prevention and risk modification.

\section{BIBLIOGRAPHY}

23andMe Inc. 2017. Learn how your DNA may affect your health: Plan for the future. Stay one step ahead. Engage in your health care. Available at: https://www.23andme.com/en-gb/health/ [Accessed 7 February 2017].

Berg, J. S., Agrawal, P. B., Bailey, D. B., Jr., Beggs, A. H., Brenner, S. E., Brower, A. M., Cakici, J. A., Ceyhan-Birsoy, O., Chan, K., Chen, F., et al. 2017. Newborn Sequencing in Genomic Medicine and Public Health. Pediatrics, 139.

Cairns, S. R., Scholefield, J. H., Steele, R. J., Dunlop, M. G., Thomas, H. J. W., Evans, G. D., Eaden, J. A., Rutter, M. D., Atkin, W. P., Saunders, B. P., et al. 2010. Guidelines for colorectal cancer screening and surveillance in moderate and high risk groups (update from 2002). Gut, 59, pp.666-689.

Collins, R., Reith, C., Emberson, J., Armitage, J., Baigent, C., Blackwell, L., Blumenthal, R., Danesh, J., Smith, G. D., DeMets, D., et al. 2016. Interpretation of the evidence for the efficacy and safety of statin therapy. The Lancet, 388, pp.2532-2561.

Di Angelantonio, E., Bhupathiraju, S. N., Wormser, D., Gao, P., Kaptoge, S., de Gonzalez, A. B., Cairns, B. J., Huxley, R., Jackson, C. L., Joshy, G., et al. 2016. Body-mass index and all-cause mortality: individual-participant-data meta-analysis of 239 prospective studies in four continents. The Lancet, 388, pp.776-786.

Flores, M., Glusman, G., Brogaard, K., Price, N. D., and Hood, L. 2013. P4 medicine: how systems medicine will transform the healthcare sector and society. Per Med, 10, pp.565-576.

Freeman-Wange, T., Cruickshank, M., and Kitchener, H. 2016. Scientific Impact Paper No. 7: Progress in Cervical Screening in the UK. London: Royal College of Obstetricians and Gynaecologists.

GBD 2015 Risk Factors Collaborators. 2016. Global, regional, and national comparative risk assessment of 79 behavioural, environmental and occupational, and metabolic risks or clusters of 
risks, 1990-2015: a systematic analysis for the Global Burden of Disease Study 2015. Lancet, 388, pp.1659-1724.

Groskop, V. 2016. Sally Phillips: Do we really want a world without Down's syndrome? In The Guardian, London. Available at: https://www.theguardian.com/lifeandstyle/2016/oct/2001/do-wereally-want-a-world-without-downs-syndrome-ds-prenatal-test [Accessed 5 February 2017].

Hamdy, F. C., Donovan, J. L., Lane, J. A., Mason, M., Metcalfe, C., Holding, P., Davis, M., Peters, T. J., Turner, E. L., Martin, R. M., et al. 2016. 10-Year Outcomes after Monitoring, Surgery, or Radiotherapy for Localized Prostate Cancer. N Engl J Med, 375, pp.1415-1424.

Hollands, G. J., French, D. P., Griffin, S. J., Prevost, A. T., Sutton, S., King, S., and Marteau, T. M. 2016. The impact of communicating genetic risks of disease on risk-reducing health behaviour: systematic review with meta-analysis. BMJ, 352, pp.i1102.

Horne, R., Bell, J. I., Montgomery, J. R., Ravn, M. O., and Tooke, J. E. 2015. A new social contract for medical innovation. Lancet, 385, pp.1153-1154.

Howard, H. C., Knoppers, B. M., Cornel, M. C., Wright Clayton, E., Senecal, K., Borry, P., endorsed by the European Society of Human Genetics, the P. G. International Paediatric Platform, the Human Genome Organisation, and the P. H. G. Foundation. 2015. Whole-genome sequencing in newborn screening? A statement on the continued importance of targeted approaches in newborn screening programmes. Eur J Hum Genet, 23, pp.1593-1600.

Human Longevity Inc. 2016. Human Longevity Inc. Advances Leadership Role in Newborn Genome Sequencing with Strategic Acquisition of Stem Cell Banking from Celgene Cellular Therapeutics: HLI to Offer Newborn Genome Sequencing Product in Combination with Stem Cell Banking. Available at: http://www.humanlongevity.com/human-longevity-inc-advances-leadership-role-innewborn-genome-sequencing-with-strategic-acquisition-of-stem-cell-banking-from-celgene-cellulartherapeutics/ [Accessed 7 February 2017].

Jacobs, I. J., Menon, U., Ryan, A., Gentry-Maharaj, A., Burnell, M., Kalsi, J. K., Amso, N. N., Apostolidou, S., Benjamin, E., Cruickshank, D., et al. 2016. Ovarian cancer screening and mortality in the UK Collaborative Trial of Ovarian Cancer Screening (UKCTOCS): a randomised controlled trial. Lancet, 387, pp.945-956.

Kotsopoulos, J., Huzarski, T., Gronwald, J., Singer, C. F., Moller, P., Lynch, H. T., Armel, S., Karlan, B., Foulkes, W. D., Neuhausen, S. L., et al. 2017. Bilateral Oophorectomy and Breast Cancer Risk in BRCA1 and BRCA2 Mutation Carriers. J Natl Cancer Inst, 109.

National Institite for Health and Care Excellence. 2008 (Updated 2016). Familial hypercholesterolaemia: identification and management (CG71). NICE Guidelines. Available at: https://www.nice.org.uk/guidance/cg71 [Accessed 7 February 2017].

Norheim, O. F., Jha, P., Admasu, K., Godal, T., Hum, R. J., Kruk, M. E., Gómez-Dantés, O., Mathers, C. D., Pan, H., Sepúlveda, J., et al. 2015. Avoiding 40\% of the premature deaths in each country, 2010-30: review of national mortality trends to help quantify the UN Sustainable Development Goal for health. The Lancet, 385, pp.239-252.

Paluch-Shimon, S., Cardoso, F., Sessa, C., Balmana, J., Cardoso, M. J., Gilbert, F., and Senkus, E. 2016. Prevention and screening in BRCA mutation carriers and other breast/ovarian hereditary cancer syndromes: ESMO Clinical Practice Guidelines for cancer prevention and screening. Ann Oncol, 27, pp.v103-v110.

Pashayan, N., Duffy, S. W., Neal, D. E., Hamdy, F. C., Donovan, J. L., Martin, R. M., Harrington, P., Benlloch, S., Amin Al Olama, A., Shah, M., et al. 2015a. Implications of polygenic risk-stratified screening for prostate cancer on overdiagnosis. Genet Med, 17, pp.789-795.

Pashayan, N., Pharoah, P. D., Schleutker, J., Talala, K., Tammela, T., Maattanen, L., Harrington, P., Tyrer, J., Eeles, R., Duffy, S. W., and Auvinen, A. 2015b. Reducing overdiagnosis by polygenic riskstratified screening: findings from the Finnish section of the ERSPC. Br J Cancer, 113, pp.1086-1093. 
PDQ Screening and Prevention Editorial Board. 2016. Prostate Cancer Screening (PDQ®): Health Professional Version. In PDQ Cancer Information Summaries [Internet]. Bethesda, MD: National Cancer Institute (US). Available at: https://www.ncbi.nlm.nih.gov/books/NBK65945/.

Pirie, K., Peto, R., Reeves, G. K., Green, J., and Beral, V. 2013. The 21 st century hazards of smoking and benefits of stopping: a prospective study of one million women in the UK. Lancet, 381, pp.133141.

Rosenthal, A. N., Fraser, L., Manchanda, R., Badman, P., Philpott, S., Mozersky, J., Hadwin, R., Cafferty, F. H., Benjamin, E., Singh, N., et al. 2013. Results of Annual Screening in Phase I of the United Kingdom Familial Ovarian Cancer Screening Study Highlight the Need for Strict Adherence to Screening Schedule. J Clin Oncol, 31, pp.49-57.

Stoffel, E. M., Mangu, P. B., Gruber, S. B., Hamilton, S. R., Kalady, M. F., Lau, M. W., Lu, K. H., Roach, N., and Limburg, P. J. 2015. Hereditary colorectal cancer syndromes: American Society of Clinical Oncology Clinical Practice Guideline endorsement of the familial risk-colorectal cancer: European Society for Medical Oncology Clinical Practice Guidelines. J Clin Oncol, 33, pp.209-217.

The NHS RAPID Project. 2016. Guides to NIPD / NIPT: Prenatal non-invasive diagnosis and testing explained. Available at: http://www.rapid.nhs.uk/guides-to-nipd-nipt/ [Accessed 6 February 2017]. 